ASTHMA

\title{
Relation between phase of the menstrual cycle and asthma presentations in the emergency department
}

\author{
B E Brenner, T M Holmes, B Mazal, C A Camargo Jr
}

Thorax 2005;60:806-809. doi: 10.1136/thx.2004.033928

See end of article for authors' affiliations

.....................

Correspondence to: Dr B E Brenner, Department of Emergency Medicine, University of Arkansas for Medical Sciences, Little Rock, AR 72205, USA;

brennerbarry@uams.edu

Received 26 August 2004 Accepted 20 May 2005
Background: The phase of the menstrual cycle is thought to influence the course of asthma in women. One recent study reported a large increase in exacerbations during the perimenstrual phase, while another found a preovulatory increase. A study was undertaken to determine the relation between phase of the menstrual cycle and acute asthma in patients presenting to the emergency department (ED).

Methods: All women aged 18-54 years presenting with a diagnosis of acute asthma exacerbation were considered for enrolment in the study. Women who were pregnant, postmenopausal, following hysterectomy, with a $>28$ day menstrual cycle or incomplete reproductive history were excluded. The 792 eligible women were classified by menstrual phase based on both date of symptom onset and date of ED visit.

Results: When classified by date of symptom onset, $28 \%$ were preovulatory (days 5 to 11 ), $25 \%$ were periovulatory (days 12 to 18), $21 \%$ were postovulatory (days 19 to 25 ), and $27 \%$ were perimenstrual (days 26 to $4 ; p=0.03$ ). When classified by date of ED visit, $28 \%$ were preovulatory, $22 \%$ were periovulatory, $22 \%$ were postovulatory, and $27 \%$ were perimenstrual $(p=0.004)$. Using either approach, there were no significant differences in demographic factors or in asthma severity of women in the various menstrual phase groups.

Conclusion: Acute asthma exacerbations do not markedly increase during the perimenstrual phase. The results support the suggestion that both preovulatory and perimenstrual phases are actual triggers of asthma exacerbation in some women, or that these two phases serve as "co-factors" that worsen other recognised triggers of acute asthma.
A sthma is a significant chronic illness in the US that has been increasing in prevalence for at least two decades. Asthma related healthcare utilisation has increased throughout this same period. For example, the annual age adjusted rate of emergency department (ED) visits for asthma has increased in the US from 57 per 10000 population in 1992 to 73 per 10000 population in $1999 .^{2}$

In studying the increase, several groups have found sex differences in the prevalence of asthma across the life cycle. ${ }^{3}$ Asthma is more common initially in boys but then becomes more common among women of reproductive age. According to the 2000 Behavioral Risk Factor Surveillance System, the prevalence of asthma is $9.1 \%$ in adult women and $5.1 \%$ in men. ${ }^{4}$ ED visits follow the same pattern, with a predominance of boys among children presenting to the ED with acute asthma ${ }^{5}$ and a predominance of women among adults. ${ }^{6}$

These life cycle differences suggest that reproductive hormones influence the development of asthma and its severity. In population based studies, rates of admissions to hospital for asthma are similar by sex in the early teenage years ${ }^{78}$ but three times higher in women than in men aged 20-50 years. This pattern continues until menopause when the incidence of asthma falls and equalises with men. ${ }^{8}$

Reproductive hormones may have beneficial or deleterious effects on women with asthma for unclear reasons. ${ }^{9}$ For example, differential effects of pregnancy on asthma are well described, with one third improving, one third remaining unchanged, and one third worsening. ${ }^{10}{ }^{11}$ Female sex of the fetus exacerbates asthma during pregnancy. ${ }^{12}$ In contrast the anti-gonadotrophic androgenic agent danazol, or agents which induce a reversible menopause, gonadotrophic releasing hormone analogues, improve even severe menstrual asthma symptoms. ${ }^{13-15}$ Asthma exacerbation during the menstrual cycle might be a marker for the subset of patients who respond adversely to reproductive hormones such as oestrogen, progesterone, or anti-gonadotrophic hormones.

As early as the 19th century physicians began considering the menstrual cycle as a possible trigger for asthma. ${ }^{16}$ The hypothesis that hormonal fluctuations during the menstrual cycle might have a role in the pathophysiology of asthma has been advanced by several investigators. ${ }^{17-23}$ The results from two previously published studies were inconsistent as to the relation between phase of the menstrual cycle and ED presentation with asthma exacerbation. ${ }^{24} 25$ This study provides a much larger patient population than previous studies. The purpose of this prospective study was to determine if there is an association between phase of the menstrual cycle and both frequency of ED visits and the severity of the asthma exacerbation.

\section{METHODS}

The study was conducted at the Brooklyn Hospital Center emergency department between January 1997 and December 1999. Patients were enrolled and interviewed 24 hours per day, 7 days per week throughout the study period by trained research assistants. All acute asthma patients were screened for inclusion. Patients were asked the date of their last menstrual period during the triage process in the emergency department, whether they were pregnant, their medications such as birth control pills, and about surgical history such as hysterectomy. No other questions were asked about their menstrual cycle. Inclusion criteria were female sex, age 18-54, physician diagnosis of acute asthma, and last menstrual period within 28 days of the ED visit. Since the cycle was idealised to 28 days, patients were sorted into their respective menstrual phase based on the range from their last menstrual period. Patients were excluded if they were pregnant, on oral contraceptives, had a surgical or 
physiological menopause, or the visit was not their first ED visit for an asthma exacerbation during the study period.

Data collected about each study subject included demographic factors, past medical history (including asthma triggers and menstrual history), current medications, compliance, and details of the current asthma episode. Measures of asthma severity included history of intubation, oral steroids, or ED visit within the past year, initial peak expiratory flow (PEF), final PEF, and proportion admitted to the hospital.

As in two earlier studies, ${ }^{24} 25$ the menstrual cycle was assumed to have a duration of 28 days and women were divided into the following four categories with day 1 considered to be the onset of menstruation: preovulatory (days 5 to 11 ), periovulatory (days 12 to 18 ), postovulatory (days 19 to 25 ), and perimenstrual (days 26 to 4 ). Unique to this study, patients were classified into a phase based on two points in time: both the day of symptom onset and the day of the ED visit. Patients were excluded from that specific analysis if the date of symptom onset was missing (9\%), or the date of symptom onset produced a cycle that was greater than 28 days or a cycle that was unable to be classified (91\%). This resulted in a difference in sample size depending on method of classification $(n=560$ for date of symptom onset, $\mathrm{n}=792$ for date of ED visit).

\section{Statistical analysis}

All analyses were performed using SPSS (Chicago, Illinois). Density of symptom onset (or ED visits) was plotted as a function of day of menstrual cycle using Splus 4.0 (MathSoft Inc, Seattle, WA). The $\chi^{2}$ goodness of fit test was used to assess the association between phase of the menstrual cycle and asthma exacerbation. We chose a priori that women would be distributed equally across the four phases of the menstrual cycle and the analysis was done to compare the actual proportions in the four phases to 0.25. All tests are two tailed with $\mathrm{p}$ values $<0.05$ considered statistically significant.

\section{RESULTS}

There were 4987 patient visits to the ED for an asthma exacerbation during the study period of which 3208 (64\%) were women. A total of 2161 women were excluded because their menstrual period was longer than 28 days $(n=522)$, surgical or physiological menopause $(n=420)$, multiple visits ( $>2$ ED visits, $n=729$ ), oral contraceptives $(n=275)$, and no menstrual date listed $(n=215)$. Of the remaining 1047

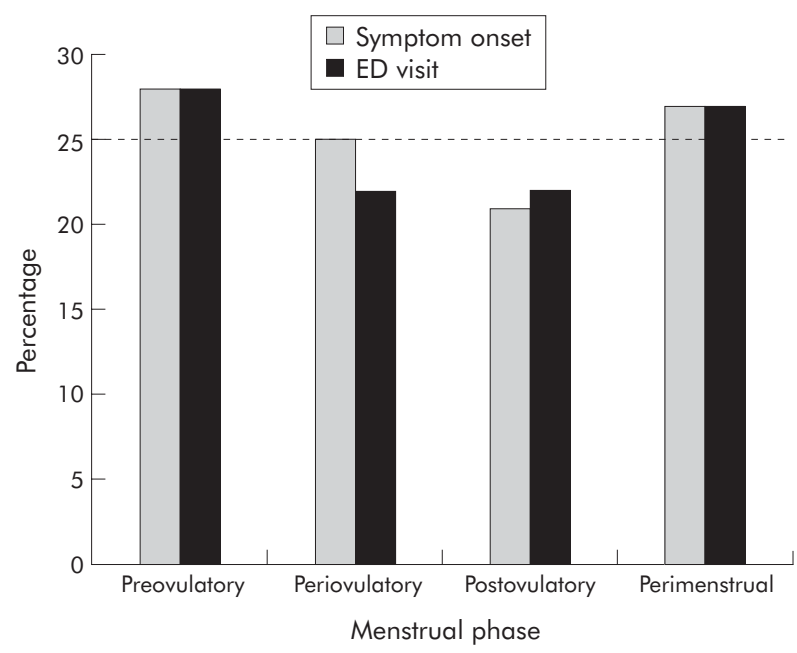

Figure 1 Menstrual phase on day of emergency department (ED) visit and day of symptom onset. women, another 255 were excluded because the present visit was the second visit to the ED for asthma during that menstrual cycle leaving 792 women with acute asthma. When the data were analysed including all ED visits (that is, more than one ED visit by a single patient), the results did not materially differ from results obtained with the dataset based on only the first ED visit. Moreover, there were no significant differences in mean age, race, median family income (based on home ZIP code), or percentage actively smoking between the study group and those excluded for the reasons cited above.

Figure 1 shows the relative proportion of ED visits according to phase of menstrual cycle when classified by either symptom onset or day of ED visit. The two methods of classification yielded similar results. Asthma exacerbations began more often during the preovulatory $(28 \%)$ and perimenstrual phases $(27 \%)$ of the cycle than in the periovulatory or postovulatory phases $(p=0.03)$. Likewise, ED visits occurred more commonly among women in the preovulatory $(28 \%)$ and perimenstrual $(27 \%)$ phases $(p=0.004)$. Figure 2 shows the density of ED asthma visits plotted as a function of the day of menstrual cycle.

Using either approach, there were no significant differences in demographic factors or in asthma severity of women according to menstrual phases. To facilitate comparison with prior studies, ${ }^{24}{ }^{25}$ table 1 shows data according to date of ED presentation with acute asthma. There were no differences between the four phases of the menstrual cycle regarding income or percentage of active smokers.

\section{DISCUSSION}

Our results show that ED visits for acute asthma are slightly more common during the preovulatory and perimenstrual phases of the menstrual cycle. This pattern was present whether the menstrual phase was estimated from the day of symptom onset or the day of the ED visit. These findings differ from Skobeloff $e a^{24}$ who found that almost $50 \%$ of 182 ED visits for acute asthma occurred during the perimenstrual phase. Our results are more consistent with the recent study by Zimmerman et $a^{25}$ on behalf of the Multicenter Airway Research Collaboration (MARC) investigators (www.emnetusa.org). They found that only $21 \%$ of 288 ED visits for acute asthma exacerbation occurred in the perimenstrual phase, whereas 33\% occurred in the preovulatory phase. In the present study involving 792 ED visits for asthma, we noted
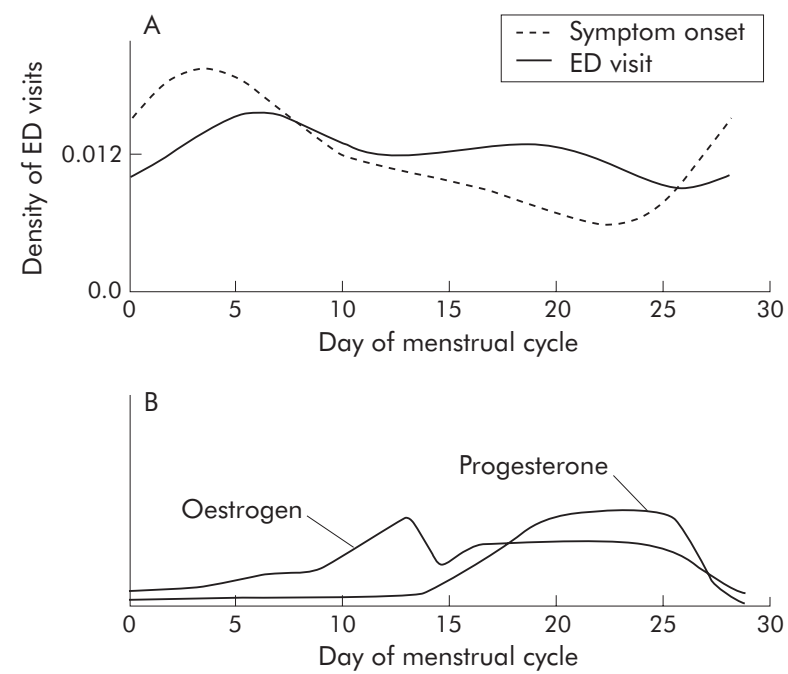

Figure 2 Emergency department (ED) visits for asthma. (A) Timing of $E D$ visit for acute asthma in relation to blood hormone levels in idealised menstrual cycle (B). 
Table 1 Demographic factors and markers of asthma severity by phase of menstrual cycle; classified by date of emergency department visit

\begin{tabular}{|c|c|c|c|c|c|}
\hline & $\begin{array}{l}\text { Preovulatory } \\
\text { (days } 5 \text { to 11) } \\
(n=225)\end{array}$ & $\begin{array}{l}\text { Periovulatory } \\
\text { (days } 12 \text { to } 18 \text { ) } \\
(n=176)\end{array}$ & $\begin{array}{l}\text { Postovulatory } \\
\text { (days } 19 \text { to } 25 \text { ) } \\
(n=176)\end{array}$ & $\begin{array}{l}\text { Perimenstrual } \\
\text { (days 26 to } 4 \text { ) } \\
(n=215)\end{array}$ & $p$ value \\
\hline Mean age (years) & 33 & 35 & 34 & 33 & 0.17 \\
\hline African-American (\%) & 75 & 75 & 76 & 76 & 0.99 \\
\hline History of intubation (\%) & 15 & 17 & 10 & 14 & 0.30 \\
\hline History of oral corticosteroids (\%) & 34 & 32 & 34 & 38 & 0.70 \\
\hline History of ED visit in the past year (\%) & 68 & 73 & 67 & 68 & 0.06 \\
\hline Initial PEF (\% pred)* & 52 & 45 & 49 & 47 & 0.11 \\
\hline Final PEF $(\% \text { pred })^{*}$ & 67 & 62 & 61 & 63 & 0.43 \\
\hline Hospitalised for index exacerbation (\%) & 14 & 13 & 18 & 9 & 0.20 \\
\hline
\end{tabular}

more modest increases in both the preovulatory $(28 \%)$ and perimenstrual $(27 \%)$ phases.

It is unclear whether the observed associations represent a hormonal effect on a certain subset of the female asthmatic population or the effect of confounding factors such as differential smoking, infection, or exposure to allergens. In the MARC study only $13 \%$ (95\% CI 9 to 18 ) of women reported reproductive factors as personal asthma triggers. ${ }^{25}$ Exactly how the normal menstrual cycle (fig l) interacts with the pathophysiology of asthma is unknown, but it seems clear that the initial ED study ${ }^{25}$ overstated the importance of perimenstrual asthma and the benefits of oestrogen. In some premenopausal women administration of oestradiol has decreased symptoms, cyclic variability in PEF, and airway reactivity. ${ }^{22}{ }^{26}$ However, in postmenopausal women, oestrogen replacement therapy can cause worsening of some asthma symptoms. ${ }^{27}$ In addition, in non-asthmatic women, oestrogen replacement therapy is associated with an increased chance of developing asthma. ${ }^{28}$ Nevertheless, comparisons of endogenous hormonal fluctuations with exogenous hormonal administration require cautious interpretation; uncritical mixing of these concepts may contribute to apparent contradictions in the literature.

Objective evaluations of airway function during the menstrual cycle in women with stable asthma have yielded inconsistent results. Some studies have noted worsening symptoms and decreases in PEF in the premenstrual and menstrual period, ${ }^{17}{ }^{18} 20$ while others found no changes in symptoms or spirometric parameters. ${ }^{29}$ Other studies found a discordance between symptoms and spirometric measurements during the menstrual cycle. ${ }^{20} 21$ In asthmatic women responsiveness to methacholine ${ }^{30}$ and adenosine monophosphate $^{31}$ increases in the luteal phase (days 24 to 28) compared with the follicular phase (days 12 to 16 ) of the menstrual cycle.

Few studies have distinguished between women who report perimenstrual worsening of asthma and those who do not. In a prospective study Shames et al ${ }^{32}$ found increased use of bronchodilators and decreased morning PEF during the perimenstrual phase in women with reported menstrual asthma compared with women without menstrual asthma. However, daily symptom scores, evening PEF, differences in forced expiratory volume in 1 second between the luteal and follicular phases, and results of methacholine challenge were not significantly different for the two groups. Agarwal and Shah found significant cyclical differences in PEF in asthmatic women who perceived a menstrual linked deterioration which was not noted in women without such deterioration..$^{23}$ In a small study Chandler et $a^{22}$ noted that, although only a small percentage of patients noted a priori premenstrual exacerbations, all had a fall in PEF or exacerbation in symptoms which improved with oestradiol.
This amelioration was not mediated, however, by $\beta_{2}$ receptors since there was no change in $\beta_{2}$ receptor density during the menstrual cycle in those with premenstrual asthma. Even if one considers that airway function worsens in the perimenstrual period, our data suggest that it does not result in more asthma exacerbations requiring acute medical care.

This study has some potential limitations. Although the sample size is relatively large, all the subjects were from one site in an urban location which may limit the generalisability of the findings. Two hundred and fifteen women were excluded because their menstrual data were missing. Only women with physiological reproductive cycle were included, so women with hysterectomies but intact ovaries were excluded as well as those on birth control pills. In addition, inaccurate reporting of the subjects' last menstrual period could have resulted in misclassification of the phase of the menstrual cycle. Only laboratory analyses could provide more accurate classification and allow the inclusion of women with menstrual cycles longer than 28 days. Finally, we note the possibility that perimenstrual and preovulatory presentations with acute asthma may have been increased by the patients' concomitant ingestion of aspirin or other analgesics that worsen asthma. We were unable to test this hypothesis, which merits further study.

In conclusion, our data do not support claims that asthma exacerbations markedly increase during the perimenstrual phase. It is possible that both preovulatory and perimenstrual phases may have some adverse impact. For specific subsets of women, these phases could be actual triggers of an asthma exacerbation or they might serve as "co-factors" that worsen other recognised triggers of acute asthma. These menstrual phase findings, together with a growing body of evidence for sex differences in asthma, ${ }^{5-9}$ support efforts to explore hormonal influences on asthma in women.

\section{Authors' affiliations}

B E Brenner, T M Holmes, B Mazal, Department of Emergency Medicine, University Hospital, University of Arkansas for Medical Sciences, Little Rock, AR, USA

C A Camargo Jr, Department of Emergency Medicine, Massachusetts General Hospital, and Channing Laboratory, Department of Medicine, Brigham and Women's Hospital, Harvard Medical School, Boston, MA, USA

Dr Camargo is supported in part by NIH grant Al-52338 (Bethesda, MD).

Competing interests: none declared.

\section{REFERENCES}

1 Camargo CA Jr, Richardson LD. Epidemiology of asthma. In: Brenner BE, eds. Emergency asthma. New York: Marcel Dekker, 1998:59-80.

2 CDC. Surveillance for asthma - United States, 1980-1999. MMWR 2002;51/SS1:1-6. 
3 Schatz M, Camargo CA Jr. The relationship of sex to asthma prevalence, healthcare utilization, and medications in a large managed care organization. Ann Allergy Asthma Immunol 2003;91:553-8.

4 CDC. Self-reported asthma prevalence among adults - United States, 2000. MMWR 2001;50:682-6.

5 Schatz M, Clark S, Emond JA, et al. Sex differences among children 2-13 years of age presenting to the emergency department with acute asthma. Pediatr Pulmonol 2004;37:523-9.

6 Singh AK, Cydulka RK, Stahmer SA, et al. Sex differences among adults presenting to the emergency department with acute asthma. Arch Intern Med 1999; 159:1237-43

7 Skobeloff EM, Spivey WH, St. Clair SS, et al. The influence of age and sex on asthma admissions. JAMA 1992;268:3437-40.

8 Becklake MR, Kauffmann F. Gender difference in airway behaviour over the human life span. Thorax 1999;54:1119-38.

9 Schatz M. Interrelationships between asthma and pregnancy: a literature review. J Allergy Clin Immunol 1999;103:S330-6.

10 Juniper EF, Newhouse MT. Effect of pregnancy on asthma: a systematic review and meta-analysis. In: Schatz M, Zeiger RS, Claman HC, eds. Asthma and immunologic diseases in pregnancy and early infancy. New York: Marcel Dekker, 1993:401-27

11 Kircher S, Schatz M, Long L. Variables affecting asthma course during pregnancy. Ann Allergy Asthma Immunol 2002;89:463-6.

12 Beecroft N, Cochrane GM, Milburn HJ. Effect of sex of fetus on asthma during pregnancy. BMJ 1998;317:856-7.

13 Gorrell GJ. Relief of asthma with danazol. Can Med Assoc J 1985; 132:100.

14 Murray RD, New JP, Barber PV, et al. Gonadotrophin-releasing hormone analogues: a novel treatment for premenstrual asthma. Eur Respir J 1999:14:966-7.

15 Blumenfeld Z, Bentur L, Yoffe N, et al. Menstrual asthma: use of a gonadotropin-releasing hormone analogue for the treatment of cyclic aggravation of bronchial asthma. Fertil Steril 1994;62:197-200.

16 Ramadge FH. Asthma, its species and complications. London: Longman, Rees, Orme, Brown, Green, Longman, 1835:41-102.

17 Hanley SP. Asthma variation with menstruation. Br J Dis Chest 1981;75:306-8.

18 Gibbs CJ, Coutts II, Lock R, et al. Premenstrual exacerbation of asthma. Thorax 1984;39:833-6.

19 Eliasson O, Scherzer HH, DeGraff AC. Morbidity in asthma in relation to the menstrual cycle. J Allergy Clin Immunol 1986;77:87-94.
20 Juniper EF, Kline PA, Roberts RS, et al. Airway responsiveness to methacholine during the natural menstrual cycle and the effects of oral contraceptives. Am Rev Respir Dis 1987; 135:1039-42.

21 Pauli BD, Reid RL, Munt PW, et al. Influence of menstrual cycle on airway function in asthmatic and normal subjects. Am Rev Respir Dis 1989;140:358-62

22 Chandler MHH, Schuldheisz S, Phillips BA, et al. Premenstrual asthma: the effects of estrogen on symptoms, pulmonary function, and $\beta_{2}$-receptors. Pharmacotherapy 1997; 17:224-34.

23 Agrawal AK, Shah A. Menstrual-linked asthma. J Asthma 1997:34:539-45.

24 Skobeloff EM, Spivey WH, Silverman R, et al. The effect of menstrual cycle on asthma presentations in the emergency department. Arch Intern Med 1996; 156:1837-40.

25 Zimmerman JL, Woodruff PG, Clark S, et al. Relation between phase of menstrual cycle and emergency department visit for acute asthma. Am J Respir Crit Care Med 2000;162:512-5.

26 Tan KS, McFarlane LC, Lipworth BJ. Modulation of airway reactivity and peak flow variability in asthmatics receiving the oral contraceptive pill. Am J Respir Crit Care Med 1997:155:1273-7.

27 Lieberman D, Kopernik G, Porath A, et al. Sub-clinical worsening of bronchial asthma during estrogen replacement therapy in asthmatic post-menopausal women. Maturitas 1995;21:153-7.

28 Barr RG, Wentowski CC, Grodstein F, et al. Prospective study of postmenopausal hormone use and newly diagnosed asthma and chronic obstructive pulmonary disease. Arch Intern Med 2004;164:379-86.

29 Weinmann GG, Zacur H, Fish JE. Absence of changes in airway responsiveness during the menstrual cycle. J Allergy Clin Immunol 1987;79:634-8.

30 Dauletbaev N, Gorijatchkina L, Ametov A, et al. Pulmonary function and bronchial hyperresponsiveness in various phases of the menstrual cycle. Am J Respir Crit Care Med 1996;153:A515.

31 Tan KS, McFarlane LC, Lipworth BJ. Loss of normal cyclical beta adrenoceptor regulation and increased premenstrual responsiveness to adenosine monophosphate in stable female asthmatic patients. Thorax 1997;52:608-11.

32 Shames RS, Heilbron DC, Janson SL, et al. Clinical differences among women with and without self-reported menstrual asthma. Ann Allergy Asthma Immunol 1998;81:65-72.

33 Nunn AJ, Gregg I. New regression equations for predicting peak expiratory flow in adults. BMJ 1989;298:1068-70.

\section{LUNG ALERT}

\section{A clinic service offering isoniazid preventive therapy to HIV infected adults reduces} the incidence of tuberculosis

$\Delta$ Grant AD, Charalambous S, Fielding KL, et al. Effect of routine isoniazid preventive therapy on tuberculosis incidence among HIV-infected men in South Africa: a novel randomized incremental recruitment study. JAMA 2005;293:2719-25

$\mathrm{T}$ his study followed 1655 HIV infected male employees of a South African gold mining company (median age 37 years) for a median duration of 22.1 months. Isoniazid preventive therapy, $300 \mathrm{mg}$ daily, was offered at clinic enrolment to 679 subjects who attended with no evidence of active tuberculosis (TB).

A total of 254 TB episodes met the study criteria. Before enrolment at the clinic the incidence of TB was 11.9 per 100 person-years, which reduced to 9.0 per 100 person-years following intervention. The adjusted incidence rate ratio for the effect of clinic enrolment on the incidence of TB was 0.68 (95\% CI 0.48 to $0.96 ; \mathrm{p}=0.03)$. The overall reduction in the incidence of TB was 38\%, and $42 \%$ in those with no prior history of TB. Failure to attain results as high as in previous studies may have been due to the fact that all the subjects who attended the clinic were assessed, irrespective of whether they received isoniazid.

Despite the reduction, the incidence rate is still high at 9 per 100 person-years. As the authors point out, long term prevention of TB needs to be considered as well as the emergence of isoniazid resistant strains. The follow up period in the study was short, and evidence suggests that the protective strategies of TB preventive therapy, particularly those without rifampicin, wane over time. Further studies to assess secondary prevention of TBalthough not currently recommended-would be interesting, particularly in view of the prolonged survival now offered by antiretroviral therapy.

A R Khan

SHO in Respiratory Medicine, Royal Free Hospital, London, UK; emonarkhan@doctors.org.uk 